\title{
Non-contact Sensation Screening of Diabetic Foot using Low Cost Infrared Sensors
}

\author{
Greg Iven, Viktor Chekh, Shuang (Sean) Luan, \\ Abdullah Mueen \\ Department of Computer Science \\ University of New Mexico \\ Albuquerque, USA \\ \{gien001, vchekh, sluan, mueen\}@unm.edu

\section{Wenyao Xu} \\ Department of Computer Science and Engineering \\ State University of New York at Buffalo \\ Buffalo, New York, USA \\ wenyaoxu@buffalo.edu
}

\author{
Peter Soliz \\ VisionQuest Biomedical LLC \\ Albuquerque, New Mexico, USA
psoliz@visionquest-bio.com \\ Albuquerque, New Mexico, USA
psoliz@visionquest-bio.com
}

\author{
Mark Burge \\ Department of Internal Medicine \\ University of New Mexico School of Medicine \\ Albuquerque, New Mexico, USA \\ mburge@salud.unm.edu
}

\begin{abstract}
We present a low-cost medical embedded system for detecting diabetic peripheral neuropathy (DPN). More specifically, we use low-cost infrared sensors to capture in a noncontact fashion the thermal response of the feet following cold stimulation. The rate of thermal regulation is then calculated based on temperature recovery and used to quantify the degree of the foot sensation. This device and methodology enables early detection of risk for DPN for in-home use or at family care clinics where fancy, expensive tests cannot be conducted.
\end{abstract}

Keywords-diabetic foot; diabetic peripheral neuropathy; infrared sensors; thermal regulation; medical embedded system

\section{INTRODUCTION}

Diabetes afflicts approximately 25.8 million Americans $(8.3 \%$ of US population) [1]. The cost for managing diabetes was 245 Billion USD in 2012. The long-term management of diabetes has become one of the greatest challenges and burdens of the US Health Care System.

In this research, we focus on the feet of people with diabetes, which are at risk for diabetic peripheral neuropathy (DPN), i.e., nerve damage. It is estimated that $50 \%$ of people with diabetes have some degree of neuropathy; $15 \%$ of them will develop a foot ulcer during the lifetime. Foot ulcers are the main cause for $85 \%$ of lower extremity amputation in patients with diabetes. The key to preventing the advanced stages of DPN is early detection and intervention. Traditional clinical techniques for screening for DPN are primarily based on sensory examination. Many such tests unfortunately present significant inter- and intra-observer variability. Studies have revealed that physicians may miss the diagnosis of diabetic peripheral neuropathy in as much as $61 \%$ of patients.

In previous work [4][5], it was proposed that diabetic neuropathy could be detected quantitatively using thermal regulation. The central idea was to use infrared imaging to measure the thermal response of the diabetic feet following cold stress. The underlying theory was that neuropathy causes impairment of blood flow in the diabetic foot [7]. Diabetic foot patients will have poor thermoregulation [6], and will recover slower to core body temperature after being cooled or warmed [2][3] compared to normal people.

In previous studies [5], the recovery of the foot to the core body temperature is captured with FLIR Model SC 305 Infrared Camera. Once the infrared video is taken, the foot on each image frame is segmented and each point on the foot plantar is registered. The temperature change for each point on the foot plantar is extracted (see Figure 1) using our bio-heat transfer model [4]: $T(t)=T_{A}+\left(T_{0}-T_{A}\right) e^{-k\left(1+Q e^{-s t}\right) t}$, where $T_{0}$ is the initial temperature; $k$ is the stationary exponent factor, $Q$ is the amplitude of regulation, $s$ is the speed of regulation, and $r(t)=k\left(1+\left(Q e^{-s t}(1-s t)\right)\right)$ accounts for thermal regulation, which is negatively correlated to thermal regulation.
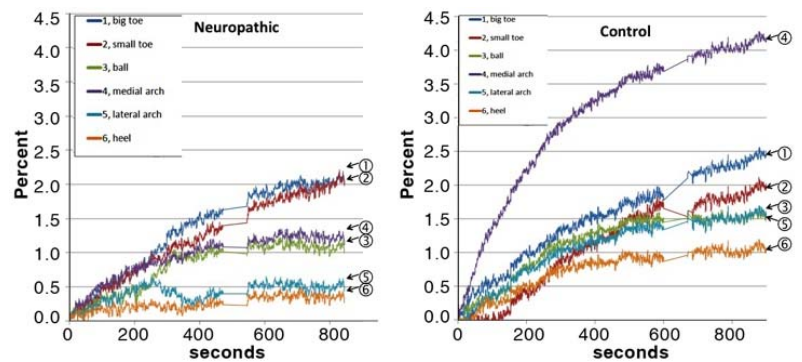

Figure 1 Differential temperature change over time of a neuropathic subject and a normal control.

The time $\Delta \mathrm{t}$ that takes $r(t)$ to its minimum, is a measure of the speed of thermal regulation of the subject's foot plantar. For an age-race-gender matched group of 8 subjects (4 normal control and 4 with confirmed diabetic peripheral neuropathy), $r(t)$ shows perfect classification. A small sample size 
statistical power analysis using binomial distribution already shows that with $95 \%$ confidence, our diagnostic software can correctly identify $47 \%$ of the diabetic foot subjects, which is an already improvement over the current $40 \%$ by the sensory testing. Since the study is done only with a small group of subjects, we believe the sensitivity of the technique will be much higher when the study is carried out on a much large scale.

\section{LOW COST INFRARED DIAGNOSIC SYSTEM}

The high cost of the camera (about $\$ 15,000$ ) means that the screening is too expensive even for clinics and can only be carried out in hospitals or diabetes care centers. Early detection and prevention of diabetic foot will require routine monitoring of the foot. Thus, the solution to the long term management of diabetic foot must be a home-oriented, patient/person centered process. It is highly demanding to develop a system that is low cost for routine in-home use.

In this paper, we present a light-weight, foot neuropathy screening system using infrared sensors. The system consists of one microcontroller (Microchip ${ }^{\circledR}$ PIC18F4550 processor), four infrared sensors (the Melexis ${ }^{\circledR}$ MLX90614-ESF-DCH infrared temperature sensors), one serial communication module and a wireless Bluetooth module. The embedded system costs about $\$ 300$, but should be significantly cheaper when mass-produced.

The Melexis ${ }^{\circledR}$ MLX90614-ESF-DCH infrared sensor is designed for non-contact temperature measurement. It is packaged in a TO-39 can with integrated low noise amplifier, 17-bit ADC and DSP unit. The field of view is 10 degree. The maximum temperature range is $-40^{\circ} \mathrm{C} \sim 125^{\circ} \mathrm{C}$ and can reach up to the resolution of $0.02^{\circ} \mathrm{C}$. In the range of $0^{\circ} \mathrm{C} \sim 50^{\circ} \mathrm{C}$, the sensor nominal accuracy is $0.5^{\circ} \mathrm{C}$. The voltage supply of the sensor is $2.6 \sim 3.6 \mathrm{~V}$, and the current consumption is about $2 \mathrm{~mA}$. When first powered on, the sensor takes 0.65 seconds to warm up, and the response time is about $50 \mathrm{~ms}$ in use. The sensor also supports a sleep mode for power saving. Under the sleep mode, the sensor pulls a negligible current of less than $6 \mu \mathrm{A}$, and it will take about $33 \mathrm{~ms}$ to wake up the sensor.

The system as designed will run on two AA batteries for at least a year with the assumption that the patient take a measurement once every two days, and each measurement takes about 15 minutes.

An Omega BB703 black body was used for the calibration of the sensors. This black body has a temperature range from ambient temperature to $100^{\circ} \mathrm{C}, 0.1^{\circ} \mathrm{C}$ resolution, and $0.3^{\circ} \mathrm{C}$ stability. We found out that the sensors were indeed within the specifications from the manufacturer. We also found that the impact of sensor to object distance is almost negligible.

We have also tested the device for 3 human subjects, two normal controls and one diabetes without diabetic foot. Figure 2 (a) shows the set up for foot sensation examination. The sensors are placed at a distance of about $5 \sim 8 \mathrm{~cm}$ away from the subject's plantar foot. Figure 2 (b) shows the temperature recovery comparisons of the 3 human subjects ( 1 diabetes and 2 normal cotrols) for one of the 4 sensors.

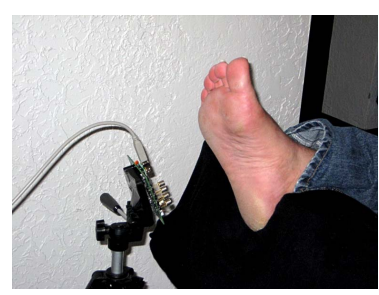

(a)

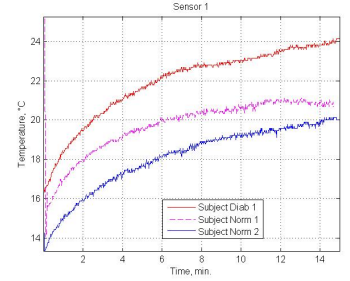

(b)
Figure 2 (a) Experimental testing of the device. (b) Temperature recovery comparisons of 3 human subjects (1 DPN subject and 2 normal controls).

\section{CONCLUSION AND FUTURE WORK}

In this paper, we present a low cost medical embedded system that can provide in-home, quantitative screening and monitoring of diabetic foot. For future research, we would like to improve the system from the following aspects:

(1) We will develop an enclosure of the system with the ability of patient foot localization.

(2) We will investigate other low-cost infrared sensors.

(3) We will carry out a larger scale patient study to verify the sensitivity and specificity of the designed embedded system. A statistical power analysis indicated that we will need to test the system for around 130 normal controls and 130 age-racegender-BMI (Body Mass Index) matched diabetic foot subjects in order to produce the statistics needed.

\section{ACKNOWLEDGMENT}

This research was funded in part by the National Institute of Diabetes and Digestive and Kidney Diseases, grant DK093192 and NSF grant CBET-0853157.

\section{REFERENCES}

[1] American Diabetes Association Fast Facts Data and Statistics about Diabetes 2013.

[2] Armstrong D, Lavery L, Wunderlich R, Boulton A. Skin temperatures as a one-time screening tool do not predict future diabetic foot complications. J Am Podiatr Med Assoc 2003; 93:443-7.

[3] Armstrong D, Sangalang M, Jolley D, Maben F, Kimbriel H, Nixon B, et al. Cooling the foot to prevent diabetic foot wounds. J Am Podiatr Med Assoc 2005;95:103-7.

[4] Chekh V, Soliz P, Barriga S, McGrew E, Kanagy N, Luan S. Novel model of thermoregulation based on control theory used to evaluate peripheral microvascular function. Experimental Biology 2013

[5] Chekh V, Luan S, Burge M, Carranza C, Soliz P, McGrew E and Barriga S. Quantitative Early Detection of Diabetic Foot. Accepted to $A C M-B C B$ 2013, Washington DC, 2013.

[6] Flynn M, Tooke J. Diabetic neuropathy and the microcirculation. Diabet Med 1995; 12:298-301.

[7] Rayman G, Williams S, Spencer P, Smaje L, Wise P, Tooke J. Impaired microvascular hyperaemic response to minor skin trauma in type 1 diabetes. Br Med J 1986; 292:1295-8 